\title{
Effective characteristics of a layered tube consisting of elastic-creeping materials
}

\author{
Tatiana Bobyleva, ${ }^{1, *}$ and Alexei Shamaev ${ }^{2,3}$ \\ ${ }^{1}$ Moscow State University of Civil Engineering, Yaroslavskoe shosse, 26, Moscow, 129337, Russia \\ ${ }^{2}$ Ishlinsky Institute for Problems in Mechanics of the Russian Academy of Sciences, 101-1, \\ Pr. Vernadskogo, 119526, Moscow, Russia \\ ${ }^{3}$ Lomonosov Moscow State University, GSP-1, Leninskie Gory, Moscow, 119991, Russia
}

\begin{abstract}
The article suggests a method for modelling the stress-strain state of layered elastic-creeping tube. This method is based on a combination of the theory of averaging and creep theory. This theory takes into account the previous stress state and its effect on the subsequent deformation period. Creep kernels for the averaged problem are found in the explicit analytical form. These formulas can be used to estimate displacement and stress fields during long-term loading of a composite tube.
\end{abstract}

\section{Introduction}

Tubes made of composite materials are widely used in construction. They are usually produced on the basis of polymers, which are characterized by phenomena of creep and relaxation. To improve the strength characteristics of pipes in their manufacture, several layers of different materials are used, which together provide high tightness and strength. The layers often differ significantly in rigidity. Examples include fibrous composites, formed by longitudinal-transverse winding. Partial differential equations which describing the behavior of such arrays contain rapidly changing coefficients. Therefore, homogenization method is used to solve such problems. This method is developed in the works [1-3]. Several examples of its application are given in [4-6]. As a rezult the problem of a heterogeneous elastic medium is replaced by a problem of a homogeneous medium for which equations have constant coefficients. These coefficients are called effective. The aim of this paper is to obtain effective properties of composite tubes with cylindrical periodicity in the microstructure. Problems for heterogeneous materials with continuously changing characteristics have been solved in [7-9].

In many composite materials, the layers that make up them have the creep property, which is the ability to deform with time at constant mechanical stresses [10-12]. For example, some problems of this type are in [13-16]. One of the most powerful approaches in solving linear elastic creeping problems consists of the reduction to a corresponding pure elastic problem. Volterra correspondence principle can be applied to the solution of such elastic-creeping problems. In the paper at first the Laplace transform was applied to all

\footnotetext{
* Corresponding author: tatyana2211@ outlook.com
} 
governing equations describing an elastic-creeping array in equilibrium. As a result, all coefficients depend on the transform parameter. Because the array has layered structure, the theory of averaging is applied to the obtained equations. Finally, the equilibrium problem for a homogeneous elastic array is obtained. We substitute previously obtained averaged (effective) coeficients in this solution and apply an inverse Laplace transform to obtain final solution.

\section{Problem specification and decision}

Let a layered tube has along its radius pairs of alternating homogeneous layers of two elastic-creeping isotropic materials (Fig.1). Let $\varepsilon$ be the length ratio of two adjacent layers to the characteristic size of the tube, and the thicknesses of each individual layer are respectively equal to: $\varepsilon_{1}=\varepsilon h$ and $\varepsilon_{2}=\varepsilon(1-h), 0 \leq h \leq 1$.

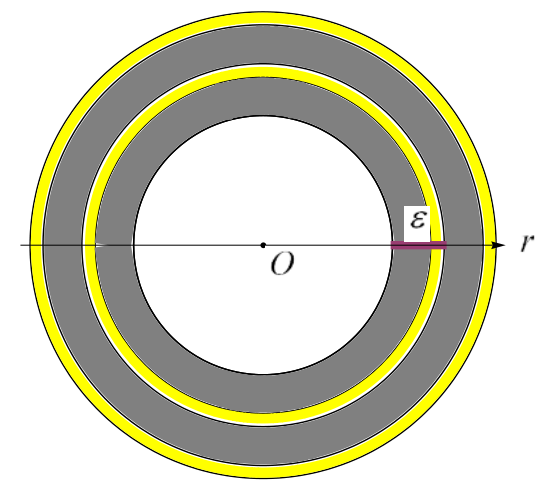

Fig. 1. The cross section of the layered tube consisting of two materials, $\varepsilon$ is the relative cell period The cylindrical coordinate system $(r ; \theta ; z)$ is used. We consider the axisymmetric case, so the displacement vector $\mathbf{u}=\mathbf{u}(r ; z)$ and stress vector $\boldsymbol{\sigma}=\boldsymbol{\sigma}(r ; z)$ do not depend on the angular coordinate $\theta$. Equilibrium equations have the following form [17]:

$$
\left\{\begin{array}{c}
\frac{\partial}{\partial r}\left[\frac{\lambda+2 \mu}{r} \frac{\partial\left(r u_{r}\right)}{\partial r}\right]+\frac{\partial}{\partial r}\left(\lambda \frac{\partial u_{z}}{\partial z}\right)+\frac{\partial}{\partial z}\left(\mu \frac{\partial u_{z}}{\partial r}\right)+\frac{\partial}{\partial z}\left[\frac{\mu}{r} \frac{\partial\left(r u_{r}\right)}{\partial z}\right]=f_{r} \\
\frac{\partial}{\partial r}\left(\mu r \frac{\partial u_{z}}{\partial r}\right)+\frac{\partial}{\partial r}\left[\lambda \frac{\partial\left(r u_{r}\right)}{\partial z}\right]+\frac{\partial}{\partial z}\left[\mu \frac{\partial\left(r u_{r}\right)}{\partial r}\right]+\frac{\partial}{\partial z}\left[r(\lambda+2 \mu) \frac{\partial u_{z}}{\partial z}\right]=r f_{z}
\end{array}\right.
$$

Vector $\mathbf{f}=\left(\begin{array}{c}f_{r} \\ r f_{z}\end{array}\right)$ means the vector of mass forces, $\lambda, \mu$ are Lame parameters, $\mathbf{u}=\left(\begin{array}{l}u_{r} \\ u_{z}\end{array}\right)$ is the displacement vector.

Equations (1) can be written in the matrix form [1]:

$$
\frac{\partial}{\partial x_{i}}\left(A^{i j} \frac{\partial \mathbf{u}}{\partial x_{j}}\right)=\mathbf{f}
$$


In (2) we designated by $x_{1}=r$ and $x_{2}=z$, and $i, j=1,2$ (Einstein convention for repeated indices is used). Matrices $A^{i j}$ have the form:

$$
A^{11}=\left(\begin{array}{cc}
\frac{\lambda+2 \mu}{r} & 0 \\
0 & \mu r
\end{array}\right), A^{12}=\left(\begin{array}{cc}
0 & \lambda \\
\mu & 0
\end{array}\right), A^{21}=\left(\begin{array}{cc}
0 & \mu \\
\lambda & 0
\end{array}\right), A^{22}=\left(\begin{array}{cc}
\frac{\mu}{r} & 0 \\
0 & r(\lambda+2 \mu)
\end{array}\right)
$$

The material of the layers that make up the pipe is an elastic-creeping one. Therefor as the main rheological relations, we take the relations of the theory of linear heredity of Boltzmann-Volterra, according to which equations (2) can be written in the form:

$$
\frac{\partial}{\partial x_{k}}\left(a_{i j k h} \delta(t)+d_{i j k h}\right) * \frac{\partial u_{j}}{\partial x_{h}}=f_{i}(\mathbf{x}, t) .
$$

Here the symbol * denotes the convolution operator:

$$
\left(d_{i j k h}(x, t) * \frac{\partial u_{j}}{\partial x_{k}}\right)=\int_{0}^{t} d_{i j k h}(x, t-\tau) \frac{\partial u_{j}(x, \tau)}{\partial x_{k}} d \tau .
$$

Natural boundary conditions are accepted. As it was said, the layers of elastic-creeping materials are assumed to be isotropic, therefore the tensors $a_{i j k h}$ in (2) and $d_{i j k h}$ in (3), respectively, have the form [11]:

$$
\begin{gathered}
a_{i j k h}=\lambda \delta_{i j} \delta_{k h}+\mu\left(\delta_{i k} \delta_{j h}+\delta_{i h} \delta_{j k}\right), \\
d_{i j k h}=-\left(D_{v}(t)-\frac{1}{3} D_{s h}(t)\right) \delta_{i j} \delta_{k h}-\frac{1}{2} D_{s h}(t)\left(\delta_{i k} \delta_{j h}+\delta_{i h} \delta_{j k}\right) .
\end{gathered}
$$

We denote here by $D_{s h}$ and $D_{v}$ the regular part of the shear and the bulk relaxation respectively, by $\delta_{i j}$ Kronecker symbol. Suppose that the amplitude of a bulk relaxation kernel is proportional to the amplitude of the shear relaxation kernel with a proportionality coefficient $k_{s}$ for each layer: $\left(D_{v}\right)_{s}=k_{s}\left(D_{s h}\right)_{s}, k_{s}$ is a constant, $k_{s}>0(s=1,2)$. Further, $D_{s h}$ is denoted by $D$.

Since this array is a layered medium all elastic modulus and relaxation kernels are periodic functions of the coordinate $\xi=\frac{r}{\varepsilon}$ ( $\varepsilon$ is the relative cell period) and are piecewise constant functions of this variable, i.e., elastic modulus and relaxation kernels have the form [16]:

$$
\lambda(\xi)=\left\{\begin{array}{l}
\lambda_{1}, \xi \in[0 ; h] \\
\lambda_{2}, \xi \in[1-h ; 1]
\end{array} \quad \mu(\xi)=\left\{\begin{array}{l}
\mu_{1}, \xi \in[0 ; h] \\
\mu_{2}, \xi \in[1-h ; 1]
\end{array} \quad D(\xi, t)=\left\{\begin{array}{l}
D_{1}(t), \xi \in[0 ; h] \\
D_{2}(t), \xi \in[1-h ; 1]
\end{array} .\right.\right.\right.
$$

In (8) $\lambda_{i}, \mu_{i}$ are Lame parameters for each layer. Let us choose creep kernel of exponential type for each layer: $D_{i}=d_{i} e^{-\alpha_{i} t}$, where $\alpha_{i}, d_{i}$ are constants, $\alpha_{i}>0, d_{i}>0,(i=1,2), t$ is the variable that specifies time, $h$ is the constant that determines the thickness ratio of layers of different materials.

We apply the Laplace transform in the time domain to the equations (1) 


$$
\tilde{f}(p)=\int_{0}^{\infty} f(t) e^{-p t} d t
$$

The result is the system of elasticity theory with a complex parameter $p$. We apply homogenization method described in [1-3] to this system, and obtain equilibrium equations with averaged ("effective") coefficients for a homogeneous medium:

$$
\frac{\partial}{\partial x_{i}}\left(\overline{\hat{A}}^{i j} \frac{\partial \mathbf{u}}{\partial x_{j}}\right)=\mathbf{f} \text {. }
$$

Then we apply the inverse Laplace transform and have the following averaged matrices $\hat{A}^{i j}$,

$$
\hat{A}^{11}=\left(\begin{array}{cc}
\hat{a}_{1111} & 0 \\
0 & \hat{a}_{1122}
\end{array}\right), \hat{A}^{12}=\left(\begin{array}{cc}
0 & \hat{a}_{1212} \\
\hat{a}_{1221} & 0
\end{array}\right), \hat{A}^{21}=\left(\begin{array}{cc}
0 & \hat{a}_{2112} \\
\hat{a}_{2121} & 0
\end{array}\right), \hat{A}^{22}=\left(\begin{array}{cc}
\hat{a}_{2211} & 0 \\
0 & \hat{a}_{2222}
\end{array}\right)
$$

and $\hat{A}^{21}=\left(\hat{A}^{12}\right)^{T}$. Elements of these matrices are effective coefficients $\hat{a}_{i j k h}$ :

$$
\begin{aligned}
& \hat{a}_{1111}=\frac{1}{L_{z} r}\left\{\frac{\sum_{n=1}^{2} e^{p_{n} t}\left[L_{1}\left(p_{n}+\alpha_{1}\right)-G_{1}\right]\left[L_{2}\left(p_{n}+\alpha_{2}\right)-G_{2}\right]}{p_{1}-p_{2}}+L_{1} L_{2} \delta(t)\right\}, \\
& \hat{a}_{1122}=\frac{r}{M_{z}}\left\{\frac{\sum_{n=1}^{2} e^{q_{n} t}\left[\mu_{1}\left(q_{n}+\alpha_{1}\right)-g_{1}\right]\left[\mu_{2}\left(q_{n}+\alpha_{2}\right)-g_{2}\right]}{q_{1}-q_{2}}+\mu_{1} \mu_{2} \delta(t)\right\}, \\
& \hat{a}_{1212}=\frac{h}{L_{z}}\left\{\sum_{n=1}^{2} \frac{(-1)^{n-1} e^{p_{n} t}\left[\lambda_{1}\left(p_{n}+\alpha_{1}\right)-j_{1}\right]\left[L_{2}\left(p_{n}+\alpha_{2}\right)-G_{2}\right]}{p_{1}-p_{2}}+\lambda_{1} L_{2} \delta(t)\right\}+ \\
& +\frac{1-h}{L_{z}}\left\{\sum_{n=1}^{2} \frac{(-1)^{n-1} e^{p_{n} t}\left[\lambda_{2}\left(p_{n}+\alpha_{2}\right)-j_{2}\right]\left[L_{1}\left(p_{n}+\alpha_{1}\right)-G_{1}\right]}{p_{1}-p_{2}}+\lambda_{2} L_{1} \delta(t)\right\}, \\
& \hat{a}_{2112}=\frac{1}{M_{z}}\left\{\frac{\sum_{n=1}^{2} e^{q_{n} t}\left[\mu_{1}\left(q_{n}+\alpha_{1}\right)-g_{1}\right]\left[\mu_{2}\left(q_{n}+\alpha_{2}\right)-g_{2}\right]}{q_{1}-q_{2}}+\mu_{1} \mu_{2} \delta(t)\right\}, \\
& \hat{a}_{2211}=\frac{1}{r M_{z}} \sum_{n=1}^{2} \frac{e^{p_{n} t}\left[\mu_{1}\left(p_{n}+\alpha_{1}\right)-j_{1}\right]\left[\mu_{2}\left(p_{n}+\alpha_{2}\right)-j_{2}\right]}{p_{1}-p_{2}}- \\
& -\frac{e^{-\alpha_{1} t} g_{1} h+e^{-\alpha_{2} t} g_{2}(1-h)}{r}+\frac{M}{r} \delta(t),
\end{aligned}
$$




$$
\begin{gathered}
\hat{a}_{2222}=\frac{(1-h) h r}{L_{z}} \sum_{n=1}^{2} \frac{(-1)^{n} e^{p_{n} t}}{p_{1}-p_{2}}\left\{\left[\lambda_{1}\left(p_{n}+\alpha_{1}\right)-2 j_{1}\right] \lambda_{1}\left(p_{n}+\alpha_{2}\right)+\frac{j_{1}^{2}\left(p_{n}+\alpha_{2}\right)}{\left(p_{n}+\alpha_{1}\right)}+\right. \\
+\left[\lambda_{2}\left(p_{n}+\alpha_{2}\right)-2 j_{2}\right] \lambda_{2}\left(p_{n}+\alpha_{1}\right)+2\left[\lambda_{1}\left(p_{n}+\alpha_{1}\right)-j_{1}\right]\left[\lambda_{2}\left(p_{n}+\alpha_{2}\right)-j_{2}\right]+ \\
\left.+\frac{j_{2}^{2}\left(p_{n}+\alpha_{1}\right)}{\left(p_{n}+\alpha_{2}\right)}\right\}+\frac{(1-h) h r}{L_{z}} \sum_{n=1}^{2} \frac{(-1)^{n} e^{-\alpha_{n} t}\left(\alpha_{2}-\alpha_{1}\right)}{\left(p_{1}+\alpha_{n}\right)\left(p_{2}+\alpha_{n}\right)}-\left(\lambda_{1}^{2}+\lambda_{1} \lambda_{2}+\lambda_{2}^{2}\right) \delta(t)- \\
-r\left[e^{-\alpha_{1} t} G_{1} h+e^{-\alpha_{2} t} G_{2}(1-h)-L\right] \delta(t) .
\end{gathered}
$$

Here $p_{1}, p_{2}$ are roots of the following quadratic equation:

$$
L_{z} p^{2}+\left[L_{z}\left(\alpha_{1}+\alpha_{2}\right)-\left((1-h) G_{1}+h G_{2}\right)\right] p+\left[L_{z} \alpha_{1} \alpha_{2}-G_{2} h \alpha_{1}-G_{1}(1-h) \alpha_{2}\right]=0
$$

with positive discriminant $D=\left[G_{2} h-G_{1}(1-h)+L_{z}\left(\alpha_{1}-\alpha_{2}\right)\right]^{2}+4 G_{1} G_{2} h(1-h)$, therefore $p_{1}, p_{2}$ there will be only real numbers.

Also in these formulas $q_{1}, q_{2}$ are roots of the following quadratic equation: $M_{z} q^{2}+\left[M_{z}\left(\alpha_{1}+\alpha_{2}\right)-\left((1-h) g_{1}+h g_{2}\right)\right] q+\left[M_{z} \alpha_{1} \alpha_{2}-g_{2} h \alpha_{1}-g_{1}(1-h) \alpha_{2}\right]=0$, which has a positive discriminant, consequently, $q_{1}, q_{2}$ will be real numbers as well. In these formulas we have denoted ( $i$ is the layer number):

$$
\begin{gathered}
L_{i}=\lambda_{i}+2 \mu_{i}, G_{i}=d_{i}\left(k_{i}+\frac{2}{3}\right), j_{i}=d_{i}\left(k_{i}-\frac{1}{3}\right), g_{i}=\frac{d_{i}}{2},(i=1,2), \\
L=L_{1} h+L_{2}(1-h), M=\mu_{1} h+\mu_{2}(1-h), L_{z}=L_{1}(1-h)+L_{2} h, M_{z}=\mu_{1}(1-h)+\mu_{2} h .
\end{gathered}
$$

\section{Conclusion}

The article presents a method of calculating stress-strain state of layered elastic creeping tubes. The theory of homogenization and the creeping theory were applied to solve this problem. Functions given the creep kernels have a form of exponential functions of two parameters. Method, proposed in this paper, allows to reduce the solution of the elasticcreeping problem of the heterogeneous array to the problem of pure elasticity with elastic modulus that depend slowly on the radius. The obtained formulas give an exact analytical solution for determination the effective parameters of elastic-creeping layered tubes. These formulas can be used to predict the behavior of tubes made of composite materials under the influence of long-term loads.

\section{References}

1. O.A. Oleynik, A.S. Shamaev, G.A. Yosifian, Mathematical Problems in Elasticity and Homogenization (Elsevier, North-Holland, 1992)

2. D.I. Bardzokas, A.I. Zobnin, Mathematical Modelling of Physical Processes in Composite Materials of Periodical Structures (URSS, Moscow, 2003)

3. E. Sanchez-Palencia, Non-Homogeneous Media and Vibration Theory (Springer, Berlin, 1980) 
4. T. Bobyleva, Procedia Engineering 153, 103 (2016)

5. M.I. Savenkova, S.V. Sheshenin, I.M. Zakalyukina, Proceedings of Moscow State University of Civil Engineering 8, 42 (2013)

6. T.N. Bobyleva, Proceedings of Moscow State University of Civil Engineering 12 (8), 863 (2017)

7. V.I. Andreev, Some problems and methods of mechanics inhomogeneous bodies (ASV Publ. House, Moscow, 2002)

8. V.I. Andreev, D.A. Kapliy, Procedia Engineering 111, 36 (2015)

9. N.Yu.Tsybina, R.A.Turusova, V.I. Andreev, Procedia Engineering 153, 51 (2016)

10. Yu.N. Rabotnov, Creep of Structural Elements (Nauka, Moscow, 1966)

11. A.A. Ilyushin, B.E. Pobedrya, Foundations of the mathematical theory of thermoviscoelasticity (Nauka, Moscow, 1970)

12. B.E. Pobedria, Mechanics of composite materials (MSU, Moscow, 1984)

13. A.S. Shamaev, V.V. Shumilova, Proceedings of the Steklov Institute of Mathematics 295, 202 (2016)

14. A.S. Shamaev, V.V. Shumilova, Proceedings of the Steklov Institute of Mathematics 295, 213 (2016)

15. T.N. Bobyleva, MATEC Web of Conferences 86, 01024 (2016)

16. T.N. Bobyleva, A.S. Shamaev, Soil Mechanics and Foundation Engineering 54 (4), 224 (2017)

17. A.E.H. Love, A Treatise on the Mathematical Theory of Elasticity (Dover Publications, New York, 1944) 